\title{
Handling Qualities of a Capsule Spacecraft during Atmospheric Entry
}

\author{
Karl D. Bilimoria ${ }^{*}$ and Eric R. Mueller ${ }^{\dagger}$ \\ NASA Ames Research Center, Moffett Field, CA, 94035
}

\begin{abstract}
A piloted simulation was conducted to study handling qualities for capsule spacecraft entering the Earth's atmosphere. Eight evaluation pilots, including six pilot astronauts, provided Cooper-Harper ratings, workload ratings, and qualitative comments. The simulation began after descending through the atmospheric entry interface point and continued until the drogue parachutes deployed. There were two categories of piloting tasks, both of which required bank angle control. In one task category, the pilot followed a closedloop bank angle command computed by the backup guidance system to manage g-loads during entry. In the other task category, the pilot used intuitive rules to determine the desired bank angle independently, based on an open-loop schedule of vertical speed, Mach, and total energy specified at several range-to-target gates along the entry trajectory. Pilots were able to accurately track the bank angle guidance commands and steered the capsule toward the recovery site with essentially the same range error as the benchmark autopilot trajectory albeit with substantially higher propellant usage, and the handling qualities for this task were satisfactory. Another key result was that the complex piloting task of atmospheric entry could be performed satisfactorily, even in the presence of large dispersions, by controlling bank angle to follow a simple open-loop schedule.
\end{abstract}

\section{Introduction}

$\mathrm{H}$ andling qualities are those characteristics of a flight vehicle that govern the ease and precision with which a pilot is able to perform a flying task. ${ }^{1}$ They are a manifestation of the interaction between various factors that influence pilot perception of how well a vehicle can be flown to accomplish a desired mission. These factors include the stability and control characteristics of the vehicle, the control systems that modify these characteristics, the inceptors (e.g., control stick or throttle lever) used by the pilot to transmit control commands, the visual cues from cockpit windows and displays/instrumentation that provide flight information to the pilot, and other cues (e.g., aural, tactile) that influence the pilot in the execution of the flying task. If a vehicle has good handling qualities, the pilot does not need to expend much effort compensating for vehicle deficiencies and can therefore reserve substantial cognitive capacity to successfully tackle anomalies or emergencies encountered in flight operations.

The effects of the above factors on handling qualities have been studied in atmospheric flight vehicles over many decades. $^{2-6}$ Reference standards for the handling qualities of both fixed-wing aircraft ${ }^{7}$ and rotary-wing aircraft ${ }^{8}$ have been developed and are now in common use. Broadly speaking, these standards define a subset of the dynamics and control design space that predicts good handling qualities for a given vehicle type and flying task. For example, the standards may specify a range of combinations of damping and natural frequency for a large aircraft during landing that corresponds to satisfactory, acceptable, and unacceptable handling qualities. They provide design engineers with guidelines to optimize the flight control system and minimize potential human-vehicle interaction issues.

At this time, no reference standards exist for spacecraft handling qualities. However, a modest body of work exists on handling qualities of piloted spacecraft. Most of this literature dates back to NASA's Apollo program, covering studies conducted on lunar landing and orbital rendezvous/docking; there are also some publications on capsule entry. ${ }^{9-12}$ The ability of pilots to successfully carry out missions with new generations of spacecraft will be determined in part by their handling qualities. Some flight operations may be fully automated, while others may be executed with a human pilot engaged in various levels of supervisory control including manual flying tasks. ${ }^{14}$

\footnotetext{
${ }^{*}$ Research Scientist, Flight Trajectory Dynamics and Controls Branch, Mail Stop 210-10, Karl.Bilimoria@nasa.gov. Associate Fellow, AIAA.

${ }^{\dagger}$ Aerospace Engineer, Flight Trajectory Dynamics and Controls Branch, Mail Stop 210-10, Eric.Mueller@nasa.gov. Senior Member, AIAA.
} 
Current NASA procedures require that human-rated spacecraft provide the capability for the crew to manually control the flight path and attitude with satisfactory handling qualities. ${ }^{15}$ These procedures require that, even for flight operations that are nominally executed in a highly automated control mode, the control architecture must provide the capability for a human pilot to switch to a manual control mode - whether due to failure of an automated system or of some component of the spacecraft. In these cases of emergency reversion to manual control, where the pilot role abruptly switches from monitoring to active control, it is important that the vehicle have acceptable handling qualities. Extensive experience from aircraft development and operations has shown that pilot-monitored automatic control systems are safer when they are designed to operate in a manner consistent with human piloting techniques. This allows for improved monitoring as well as graceful reversion to manual control in the event of failures in the automatic control system. It is therefore desirable for spacecraft designers to assess early in the design cycle what the handling qualities will likely be, and to adjust their design if necessary to ensure that appropriate levels of handling qualities are available for both nominal and off-nominal operations.

An effort to develop design guidelines for spacecraft handling qualities was initiated by NASA in 2007 . A comprehensive set of guidelines should cover all classes of spacecraft and phases of flight; however, programmatic considerations made it necessary to focus initially on a few specific and relevant aspects. Lessons learned from aircraft and spacecraft handling qualities assessments over the past several decades were compiled. ${ }^{16}$ Piloted simulation studies of lunar landing ${ }^{17-19}$ and Earth orbit docking were conducted. ${ }^{20-22}$ This paper covers an initial study on handling qualities for capsule entry, evaluating manual backup options after a failure of the automatic entry guidance/control system.

\section{Capsule Entry Operations}

For a spacecraft returning to Earth, the atmospheric entry phase of flight begins when it enters the atmosphere at approximately $400,000 \mathrm{ft}$ altitude and $17,000 \mathrm{mph}$, following a deorbit burn. The subsequent trajectory of the spacecraft is governed by gravitational and aerodynamic forces - there are no propulsive forces. The aerodynamic forces acting on the spacecraft can be varied by reorienting the vehicle in the atmosphere using control surfaces, reaction control system (RCS) jets, or both. This in turn modifies the trajectory, enabling the auto/human pilot to steer the vehicle to the designated land/sea recovery site.

Unlike winged spacecraft such as the Space Shuttle, capsule spacecraft generally do not have aerodynamic control surfaces. Typically, the aerodynamic forces acting on a capsule are varied by using RCS jets to reorient the vehicle in the atmosphere. During hypersonic flight, winged spacecraft have a lift-to-drag ratio, (L/D), on the order of 1 ; for capsule spacecraft the corresponding value is on the order of 0.3 so the capability for trajectory control is correspondingly less. Although capsules are generally axisymmetric in shape, they can generate some blunt-body lift at non-zero angles of attack (see Fig. 1), with (L/D) $\sim 0.3$ attained at roughly 20 deg angle of attack during hypersonic flight. However, the capsule must be trimmed to hold that angle of attack. This is generally accomplished (as in Apollo capsules) by precisely positioning the capsule center-of-mass (c.m.) away from the axis of symmetry so that the line of total aerodynamic force (i.e., resultant of lift and drag forces at the center-of-pressure (c.p.)) passes through the c.m. as shown in Fig. 1. The flight control system provides rate damping in the pitch and yaw axes to compensate for small disturbances. From a practical point of view, it is easier to offset the c.m. toward the equipment compartment rather than the crew compartment, and the resulting trim orientation of the capsule puts the crew compartment "upside down" as shown in Fig. 1. From the crew's perspective, the spacecraft is moving "backwards" because the ablative heat shield, which is located behind the crew, must point forward for capsule entry.

The mechanism for trajectory control during entry is to tilt the lift vector relative to the local vertical. This corresponds to a rotation of the vehicle about the air-relative velocity vector and is accomplished by applying a control moment about both the roll and pitch body axes. The rotation angle about the velocity vector is the bank angle $\mu$ (see Fig. 1); it determines the component of lift that opposes the gravitational force along the local vertical, and hence influences the acceleration along the local vertical given by:

$$
\ddot{h} \approx \frac{L \cos \mu}{m}+\frac{V^{2}}{R_{E}+h}-g
$$

where $m$ is the vehicle mass, $V$ is the vehicle speed, $R_{E}$ is the radius of the Earth, $h$ is the altitude above Earth, and $g$ is the acceleration due to Earth gravity. The rate of change of vehicle speed $(\dot{V} \approx-D / m$ ) depends primarily on the drag force, which is a function of the airspeed and the local atmospheric density which decreases with altitude. The magnitude of the "eyeballs in" g-load experienced by the crew depends primarily on the drag force. Consider an 
entry trajectory with lift vector up $(\mu=0)$. In this case, the lift force opposes the gravitational force to the maximum extent possible, and the resulting altitude profile is such that the capsule spends more time in the higher altitudes where the atmosphere is less dense. This results in a lower average drag force, which in turn results in the longest down-range distance and the lowest g-loads. Conversely, an entry with lift vector down ( $\mu=180 \mathrm{deg})$ results in the shortest down-range distance and the highest g-loads. Bank angle control allows adjustments to the down-range distance, but any bank angle other than zero or $180 \mathrm{deg}$ will result in a side force on the capsule. Adjusting bank angle for down-range control, therefore, has the side effect of providing a cross-range distance. This cross-range distance builds up over time and can be adjusted with periodic bank reversals as necessary. The resulting targeting accuracy can be impressive; for example, Apollo 10 splashed down within a couple of miles of its recovery ship.

During nominal entry operations, the desired bank angle is determined by the primary entry guidance system and then executed by the flight control system which fires the appropriate RCS jets to properly orient the vehicle; the crew's function is to monitor the automated systems. The guidance system computes the desired bank angle (including bank reversals) using an algorithm that considers operational performance factors such as range-to-target, g-load profile, heating loads, and RCS propellant usage. If the primary guidance system fails, then guidance may revert to a backup system. For example, a "loads-managed" guidance system can compensate for limited/degraded navigation data by using a simpler algorithm whose primary focus is on keeping the g-loads profile within human endurance limits. Although it adjusts bank angle to compensate for disturbances, it may not perform bank reversals (to keep the guidance algorithm simple), which results in substantial cross-range errors. As a final backup capability (for example, if the loads-managed guidance system also fails) the spacecraft can be placed in a "ballistic mode" by simply spinning the capsule about its axis of symmetry at a rate of $10-15 \mathrm{deg} / \mathrm{s}$ with no capability to control the resulting trajectory. This mode results in very high g-loads and an uncontrolled (ballistic) trajectory. For example, in recent years, two separate Soyuz spacecraft had to make ballistic entries as a result of system malfunctions, landing hundreds of miles short of their recovery site. Also, ballistic entry was the nominal flight mode for Mercury capsules. Although all three types of entry trajectories described above can be flown automatically by the flight control system which fires the appropriate RCS jets to provide the desired vehicle attitude, the capability generally exists for the pilot to manually fly these types of entry trajectories.
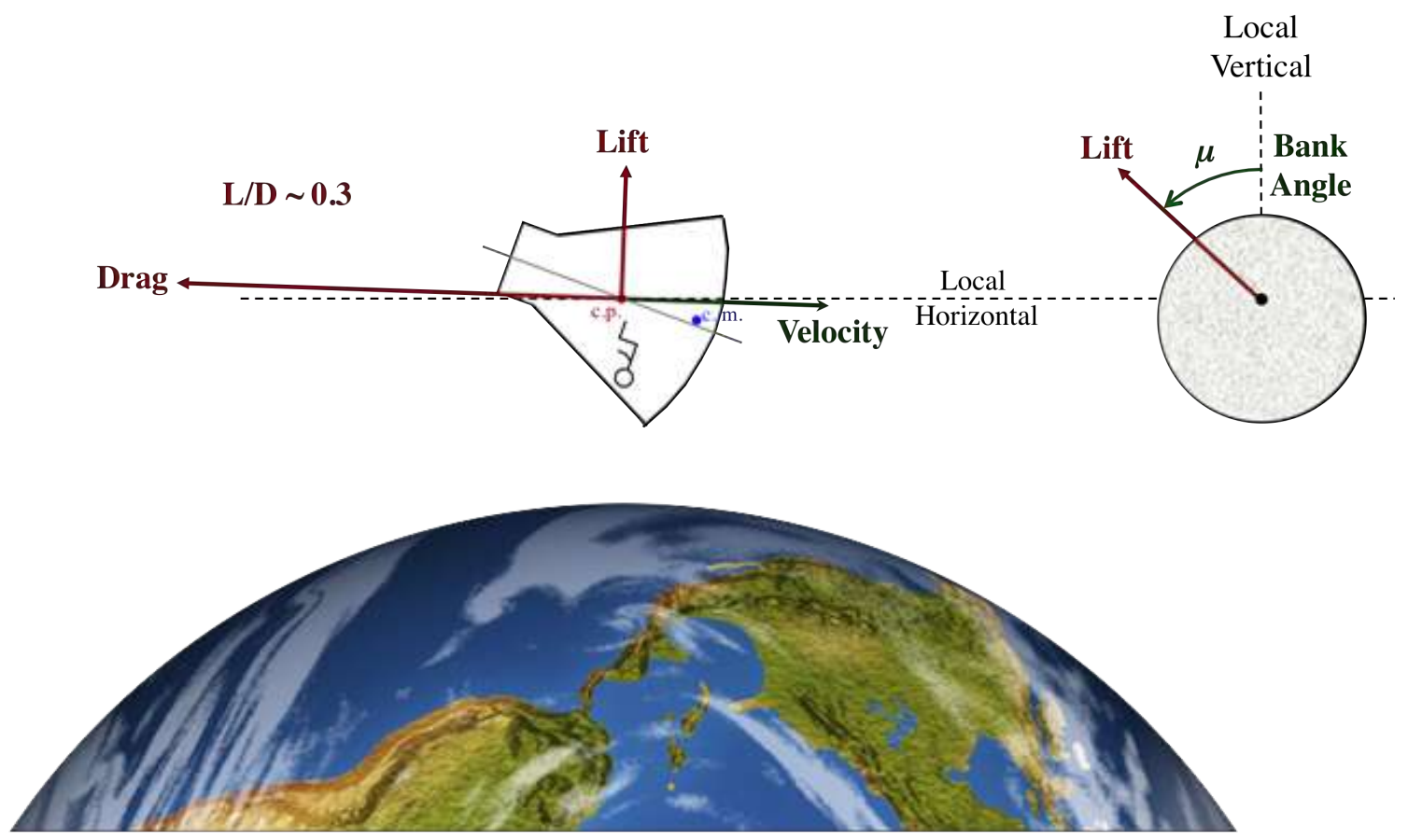

Figure 1. Illustration of capsule entry dynamics 


\section{Experiment Design}

The principal objective of this experiment was to evaluate capsule handling qualities during atmospheric entry under manual control with different guidance options. The flight segment under study was from the entry interface (EI) point to the drogue parachute deployment point. In this simulation, the target splashdown site was located roughly 200 miles west of San Diego, California. The EI point was approximately 1,800 nmi south-west of the recovery site at $400,000 \mathrm{ft}$ altitude; with an EI speed of $16,950 \mathrm{mph}$, the time to drogue deployment was about 10 min for the simulated trajectory.

Entry trajectories are influenced by many environmental and vehicle parameters such as atmospheric conditions (pressure, temperature, winds), vehicle aerodynamics and mass properties, RCS jet properties, and navigation errors. The simulation featured two scenarios, characterized by nominal and highly dispersed values of many parameters including those mentioned above.

\section{Entry Performance}

Usage of the Cooper-Harper handling qualities rating scale ${ }^{1}$ involves an assessment of pilot compensation to achieve a desired or adequate level of pilot-vehicle performance. The key operationally relevant performance parameters for entry trajectories are g-loads, propellant consumed, and range-to-target at drogue deployment. In this experiment, the g-loads constraint placed an upper limit on the time of exposure to sustained loads in excess of $3 \mathrm{~g}$, defined by a linear $\log -\log$ relationship between the points $(3 \mathrm{~g}, 100 \mathrm{~s})$ and $(10 \mathrm{~g}, 10 \mathrm{~s})$. There was an additional constraint on instantaneous g-load, with a limit of $8 \mathrm{~g}$ for desired performance (10g for adequate performance). Based on the results of autopilot and human-pilot test runs with different guidance configurations, the propellant usage limit was set to $90 \mathrm{lbm}$ for desired performance (120 lbm for adequate performance). Limits on final rangeto-target are discussed below.

\section{Piloting Tasks}

The simulation began in fast-time (i.e., non-real-time) mode with automatic control of the trajectory; it switched to real-time mode after about 3 minutes of simulation time ( $\sim 15$ seconds clock time) when the g-load built up to a value of approximately 0.17 . Shortly thereafter, when the g-load reached a value of 0.2 , the pilot took manual control; at this point the capsule was $940 \mathrm{nmi}$ from the recovery site. The pilot continued flying the spacecraft until drogue deployment.

The automatic control system provided rate damping in the pitch and yaw axes by firing the appropriate RCS jets. The pilot commanded a bank angle rate using a rotational hand controller (RHC). The maximum bank angle rate command was $20 \mathrm{deg} / \mathrm{s}$ at full displacement of the RHC, and at lower displacements it was proportionately less with some nonlinear shaping. The pilot command was converted by a flight control system mixer into the appropriate roll-rate and yaw-rate commands. These body rate commands were fed to the RCS jet firing logic which used one RCS jet to correct small bank-rate errors $(<2 \mathrm{deg} / \mathrm{s})$ and two RCS jets to correct larger bank-rate errors. The firing logic provided the commanded bank angle rate within a dead-band of $\pm 0.5 \mathrm{deg} / \mathrm{s}$. By making small pulselike RHC inputs, the pilot could adjust the bank angle to the desired value.

There were two types of flying tasks: (1) bank angle guidance tracking for loads-managed entry, and, (2) flight parameter (vertical speed, Mach, energy) schedule following for targeted entry - there were two variants of this task.

\section{Loads-Managed Entry Task}

The pilot tracked closed-loop bank angle commands generated by the backup loads-managed guidance system whose primary objective was to keep the g-loads profile within human endurance limits. The piloting task was to correct the error between the commanded and actual bank angles, using an error needle on the cockpit display (details in next section). The guidance algorithm computed the commanded bank angle by searching a 5 deg grid, and the actual bank angle had some drift due to aerodynamic moments; hence the error signal typically featured gradual variations separated by occasional jumps on the order of $5 \mathrm{deg}$. The error signal in the segments between these jumps is referred to as the steady-state error.

For this piloting task, the relevant performance parameter for the handling qualities assessment was steady-state error between commanded and actual bank angles. These error bounds were determined from fast-time Monte Carlo simulations with a simple (lag and bias) pilot model; this analysis showed that a bank angle error limit of 6 deg provided g-loads, final range-to-target and propellant usage that were within operationally acceptable bounds from the benchmark autopilot values. Accordingly, the bank angle tracking error limit was set at 6 deg for desired performance (10 deg for adequate performance).

A key goal of the piloted simulation was to determine if the operationally relevant performance parameters (g-loads, final range-to-target and propellant usage) would have acceptable values if the manual piloting task was 
simply to keep the steady-state bank angle errors within the specified limits. The performance goals for g-loads and propellant usage were as described in the previous sub-section. The benchmark autopilot runs resulted in final range-to-target values on the order of $60 \mathrm{nmi}$; hence the performance goal was a range error less than $70 \mathrm{nmi}$. The reason for the large range error is that were no bank reversals in this backup loads-managed guidance system's commands, resulting in substantial cross-range error at drogue deployment.

\section{Targeted Entry Task}

The objective of this exercise was to evaluate the feasibility, in the absence of any closed-loop guidance, of manually steering the capsule close to the target site while meeting the operational constraints of g-load profiles and propellant budgets. In the absence of bank angle guidance commands for this task, the pilot used simple intuitive rules to determine the desired bank angle independently, based on an open-loop schedule of certain flight parameters specified at six range-to-target gates along the entry trajectory. These schedules were designed using a reference trajectory generated by the autopilot following commands from the primary entry guidance system for the nominal scenario. The flight parameters in the schedule were vertical speed, Mach, and total energy (potential plus kinetic). There were two types of schedule: a schedule of vertical speed only (called the h-dot schedule), and the full schedule of vertical speed, Mach, energy. These schedules were presented on the cockpit display (details in next section). The pilot monitored the evolution of the appropriate flight parameter(s) and adjusted the bank angle using intuitive rules of physics: raise the lift vector if sinking too fast and/or energy decreasing too rapidly; lower lift vector if sinking too slow and/or energy decreasing too slowly. Bank reversals were executed at four gates in the schedule to reduce cross-range error. A key requirement of this task is to get as close as possible to the recovery site target; hence it is called the targeted entry task.

For this task, the performance parameters for the handling qualities assessment were g-loads, final range-totarget and propellant usage. The adequate performance limit for final range-to-target was set equal to the performance goal of the loads-managed entry task (i.e., $70 \mathrm{nmi}$ ), and the desired performance limit was set to $50 \%$ of the adequate limit (i.e., $35 \mathrm{nmi}$ ). The desired//adequate performance limits for g-loads and propellant usage were as described in the previous sub-section

\section{Experiment Matrix}

The principal objective of this experiment was to evaluate capsule handling qualities during atmospheric entry for a loads-managed entry task of following closed-loop bank angle guidance and a targeted entry task of following two types of open-loop schedule (h-dot and full). For each of these three tasks there were two scenarios corresponding to nominal and highly dispersed values of key parameters such as atmospheric conditions, capsule aerodynamics, c.m. location, RCS jet properties, and navigation errors. The resulting six configurations of the experiment matrix are depicted in Table 1.

\begin{tabular}{|c|c|c|c|}
\hline $\begin{array}{c}\text { Flying Task } \rightarrow \\
\text { Scenario Parameters }\end{array}$ & $\begin{array}{c}\text { Loads } \\
\text { Managed } \\
\text { Entry }\end{array}$ & \multicolumn{2}{|c|}{ Targeted Entry } \\
\hline Nominal & $\checkmark$ & $\checkmark$ & $\checkmark$ \\
\hline Dispersed & $\checkmark$ & $\checkmark$ & $\checkmark$ \\
\hline
\end{tabular}

Table 1. Experiment matrix

\section{Evaluation Pilots}

Eight experienced test pilots served as evaluation pilots for this experiment; each had several thousand flight hours in a variety of fixed and rotary wing aircraft. Two were NASA pilots with decades of aircraft flight test and simulation experience. Six were retired NASA pilot astronauts from the Space Shuttle program with a combined space flight experience of 11 missions as Pilot and 14 missions as Commander. All the astronauts had piloted atmospheric entry in a winged spacecraft (Space Shuttle) and one astronaut had also experienced atmospheric entry in a capsule spacecraft (Soyuz). Each pilot was available for about 8 hours, and this time constraint was incorporated into the experiment design. 


\section{Training Procedures}

Upon arrival, pilots received a detailed briefing on the experiment background and objectives, flying task, control system, test matrix, and data collection procedures. Including discussion time with the experimenters, this session lasted approximately one hour. This was followed by a training and familiarization session (about $1.5 \mathrm{hr}$ ) in the simulator cockpit, where pilots practiced the flying task for various representative configurations drawn from the test matrix until they felt comfortable that most of the learning curve was behind them.

\section{Data Collection Procedures}

Each pilot encountered the various experiment configurations in a different sequence and was not told whether the experiment scenario was nominal or dispersed. For each test configuration, the pilot flew two formal evaluation runs with an option for a third run if necessary (e.g., significant difference in performance across the first two runs). At the end of each run, relevant performance data were displayed to the pilot and experimenters.

In handling qualities experiments, pilots are typically asked to make a composite assessment of the overall performance across all formal evaluation runs for a test configuration. This assessment takes into account not just the quantitative evaluation of the performance at the end point (e.g., drogue deployment) but also a qualitative evaluation of the manner in which the vehicle gets to the end point. This overall assessment of desired, adequate, or inadequate performance is utilized for traversing the decision tree in the Cooper-Harper chart. ${ }^{1}$ Pilots use the Cooper-Harper scale to assign handling qualities ratings from 1 (best) to 10 (worst) based on their assessment of task performance and required compensation. It is an ordinal scale, which means for example that the difference between ratings of 1 and 2 is not the same as the difference between ratings of 3 and 4 . Ratings of 1,2, and 3 on the Cooper-Harper scale correspond to Level 1 handling qualities, which are a general requirement for normal operations of flight vehicles. Desired performance is necessary, but not sufficient, for Level 1 ratings. Ratings of 4 , 5 , and 6 correspond to Level 2 which may be acceptable for some off-nominal conditions, and ratings of 7, 8, and 9 correspond to Level 3 which is acceptable only for transition to a safe mode after a major failure or disturbance.

After making a composite assessment of the overall performance across the formal evaluation runs for a test configuration, pilots assigned a handling qualities rating for that test configuration. The pilots also assigned ratings for each of the six components of the NASA Task Load Index. ${ }^{27}$ These six components were: physical demand, mental demand, temporal demand, performance, effort, and frustration. The relative weighting of these six components for the flying task was determined by a pilot questionnaire at the end of the experiment. Workload ratings on the Bedford scale ${ }^{28}$ were also assigned by the pilots. As appropriate, pilots provided qualitative comments about the test configuration they had just evaluated. All pilot commentary was recorded on electronic media while the experimenters separately noted key comments and observations.

After all test configurations had been evaluated, there was a debrief session. The pilots filled out a one-page questionnaire designed to elicit high-level comments on cockpit displays, out-the-window displays, guidance cues, control response, and experiment design. This was followed by a discussion with the experimenters on the pros and cons of the various test configurations.

\section{Simulation Environment}

The experiment was conducted on the Vertical Motion Simulator (VMS) at the NASA Ames Research Center. The VMS is a large-travel motion simulator ${ }^{29}$ that has been used for numerous handling qualities evaluations. ${ }^{30}$ Sixdegree-of-freedom simulator motion was utilized for the experiment. A single pilot seat was installed in the center of the simulator cab, with a researcher/observer seat immediately aft of the pilot seat. A three-axis rotational hand controller (RHC) was installed on the right side of the pilot seat, and it was the only means of pilot input for this experiment. Although a three-axis translational hand controller (THC) was installed on the left side of the pilot seat, it was not used in this experiment, consistent with operating procedure for the entry piloting task. An illustration of the cockpit layout including the two control inceptors is shown in Fig. 2.

A simulated view of the Earth and sky was projected on a set of three wide-angle collimated color displays with an image resolution of $1 / 2$ pixel per arcmin. The window display had a large field of view: 48 deg vertical and 120 deg horizontal. Window masking was not used in the simulator cockpit and therefore the entire field of view was available to the pilot. This is not representative of actual operations where the pilots have only a limited field of view through small windows. However, the entry piloting task in this experiment was primarily a head-down task, and the pilot's attention was focused primarily on the cockpit instrumentation; the view outside the cockpit was used only for general situational awareness. 
The cockpit console had three 6.5-inch color flat panel displays. The center panel was the primary flight display (see Fig. 3). The upper half of this panel displayed an Attitude Director Indicator (ADI) and tapes showing Mach number, angle of attack, altitude, and vertical speed (h-dot). Key parameters displayed in the lower half of the panel were g-load, percentage of RCS propellant remaining, and current range-to-target. The blue triangle on the ADI indicated the current bank angle; for example, if the indicator was at the top of the ADI then the lift vector was pointing up (away from the Earth's surface) corresponding to zero bank angle. The yellow diamond and magenta needle were displayed only for the loads-managed entry task. The diamond indicated the bank angle guidance command, while the needle indicated the error between the commanded and actual bank angles. Pilots typically used the needle to correct bank angle errors under $10 \mathrm{deg}$. If the error was larger than $10 \mathrm{deg}$, the needle was caged and pilots used the triangle and diamond symbology to attenuate the error. The left panel displayed time histories of key flight parameters (g-load, bank angle, bank angle rate) at the end of the simulation run (see Fig. 4); it was blank during the run. The right panel served as a multifunction display. For the loads-managed entry task, this panel was blank during the simulation run. For the targeted entry task, it displayed data for range, vertical speed, Mach, and total energy, with the first data row showing current values and the data second row showing desired values at the next gate; current values of down-range and cross-range were shown at the bottom of the panel (see Fig. 4). At the end of the run, it displayed key parameters such as maximum g-load and position coordinates at drogue deployment; for the targeted entry task it also displayed (on a second page accessed by a toggle button) errors in vertical speed, Mach, and total energy at the schedule gates.

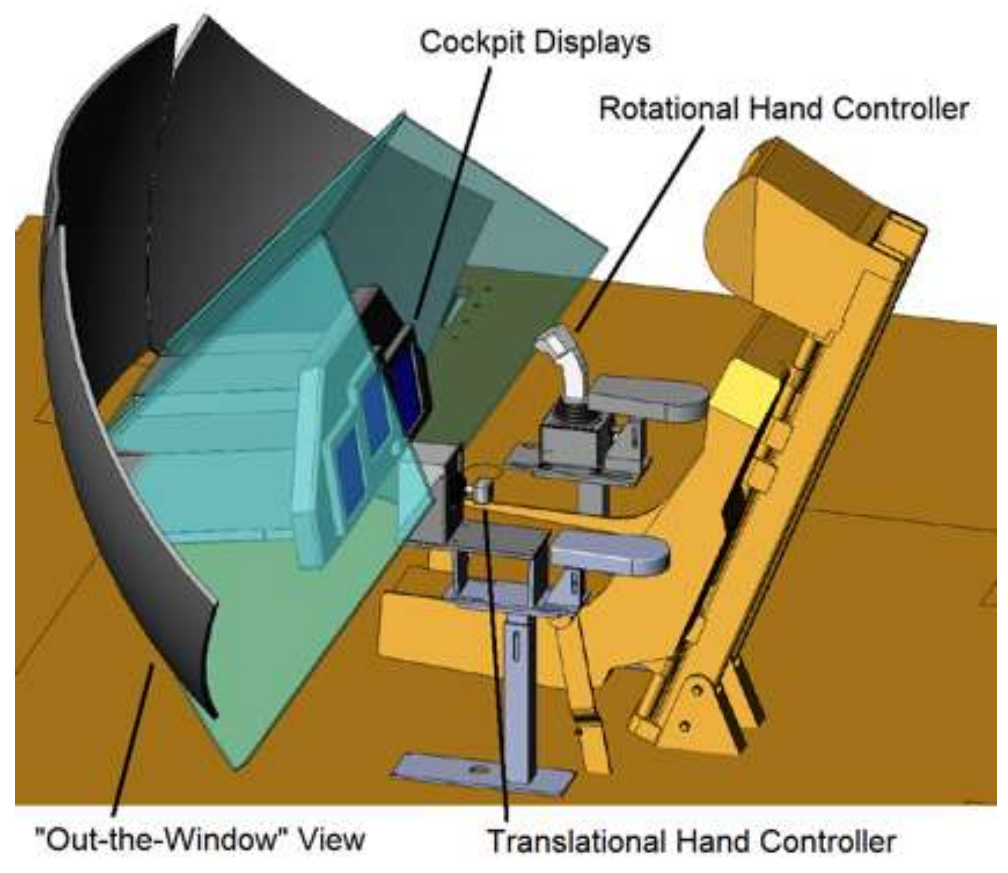

Figure 2. Simulator cockpit layout

\section{Dynamics and Control Model}

The simulation used an early version of the Orion vehicle model developed by NASA and Lockheed-Martin using the Advanced NASA Technology Architecture for Exploration Studies (ANTARES) software environment. The ANTARES simulation supports a wide variety of guidance, navigation and control engineering analyses. ${ }^{24,25}$ The VMS staff developed a software process to integrate the ANTARES model with the local simulation environment. A description of the Orion entry performance and mission design can be found in Ref. 26, while Ref. 27 provides a description of the Orion entry simulation in ANTARES. The results reported in this paper do not constitute a formal evaluation of the actual Orion vehicle design; the goal of this study was to gain insight into entry handling qualities of the capsule class of spacecraft, using a preliminary Orion vehicle model as a means to that end. 


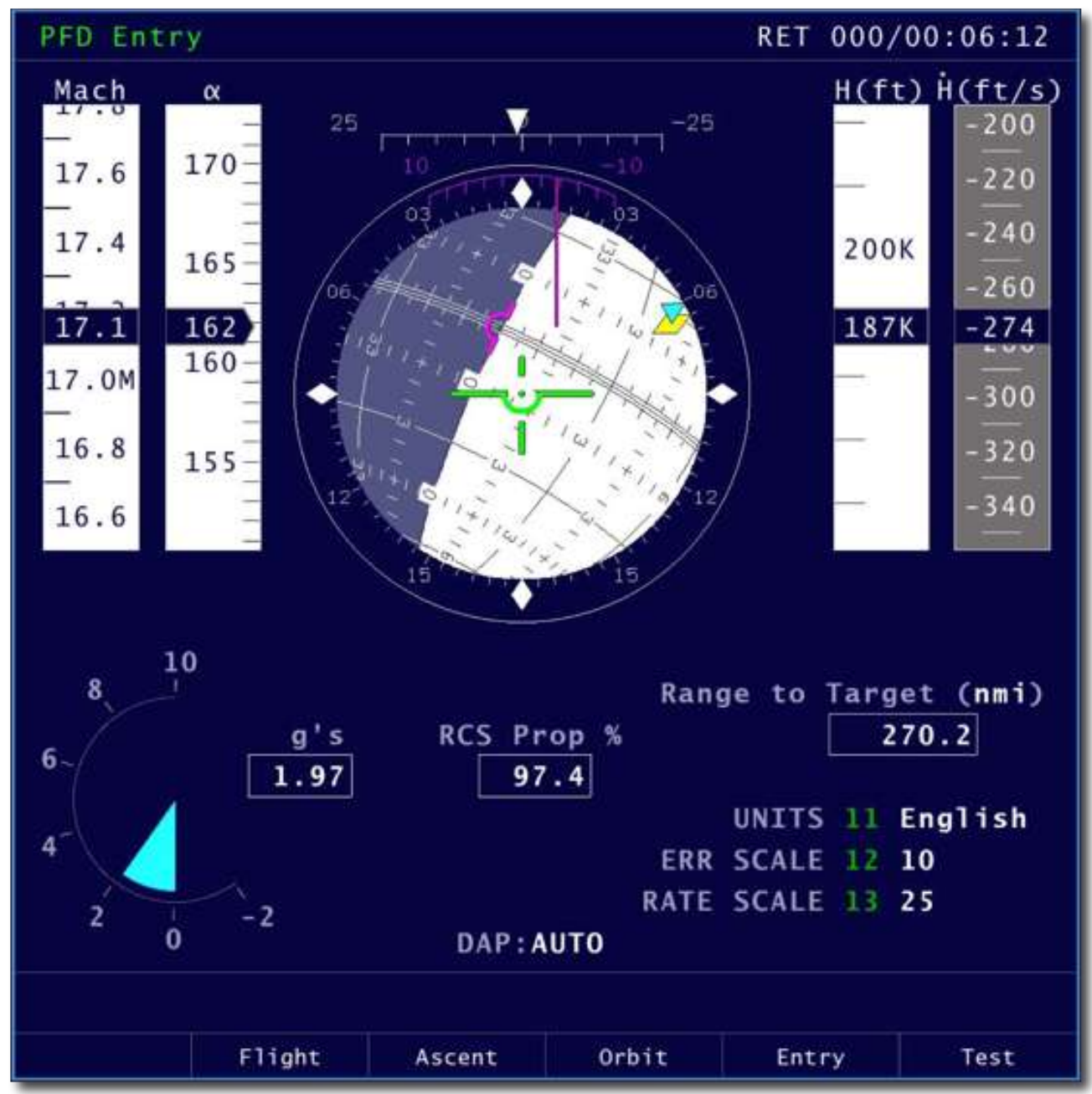

Figure 3. Primary flight display
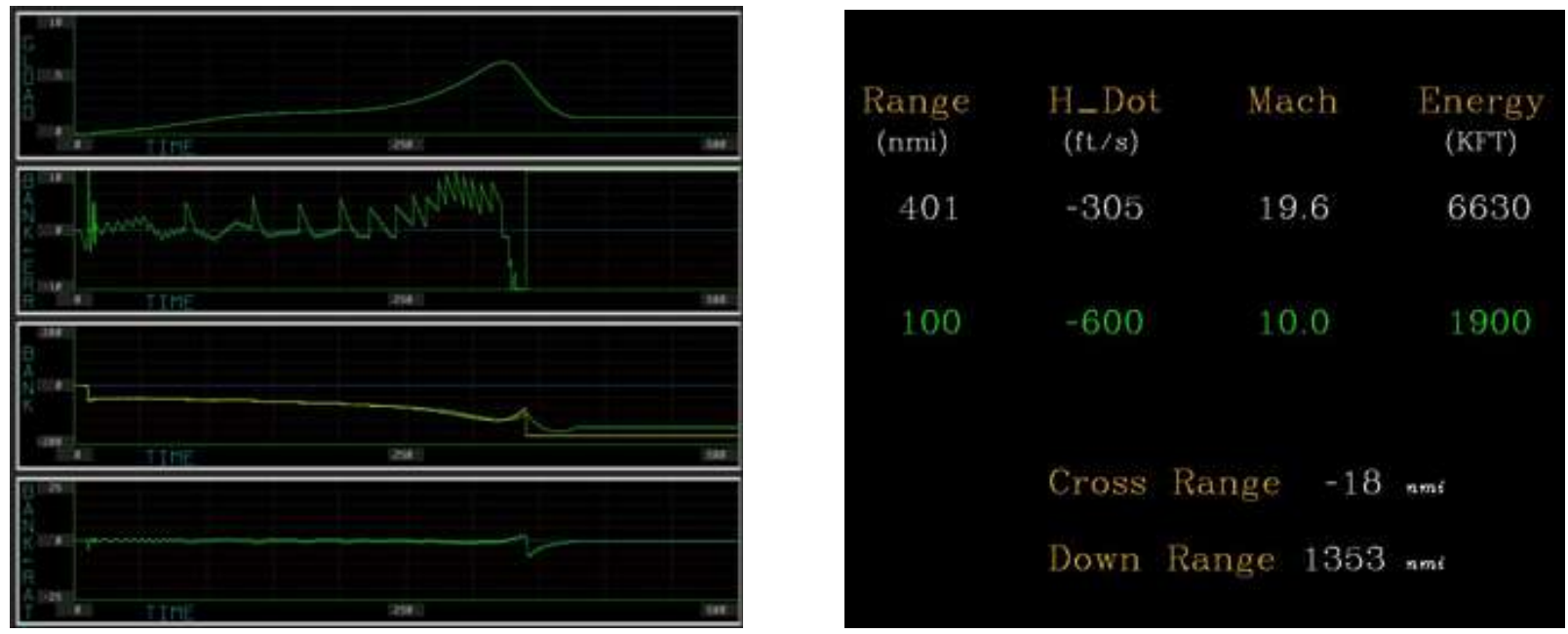

Figure 4. Auxiliary flight displays 


\section{Results}

The data collection period in the VMS was 8 - 19 June 2009. The eight evaluation pilots provided CooperHarper ratings, NASA Task Load Index (TLX) ratings, Bedford workload ratings, and specific comments for each of the experiment configurations. They also provided their overall impressions of the manual entry tasks. For each run, time histories of numerous simulation variables were recorded along with key performance parameters at drogue deployment. A total of 94 formal evaluation runs were made during this experiment. This section presents qualitative and quantitative results obtained from these runs.

\section{Task Performance}

The performance parameters of interest for this experiment were: g-loads, range-to-target at drogue deployment, propellant usage, and bank angle tracking error (for loads-managed entry task only). The results showed that, for all formal evaluation runs, g-loads were within specified human endurance limits for both peak value and exposure time.

Range error and propellant usage for all 94 formal evaluation runs, categorized by experiment configuration, are presented in Fig. 5; the circled data points indicate autopilot data. A detailed explanation of these results is provided below.

\section{Loads-Managed Entry Task}

For this task, the only performance parameter for the handling qualities assessment was steady-state bank angle error between commanded and actual values, where desired and adequate performance bounds corresponded to error magnitude less than $6 \mathrm{deg}$ and $10 \mathrm{deg}$ respectively. These error bounds were determined from fast-time Monte Carlo simulations with a simple (lag and bias) pilot model; this analysis showed that a bank angle error limit of 6 deg provided range-to-target, g-loads and propellant usage that were within operationally acceptable bounds from the benchmark autopilot values. A key goal of the piloted simulation was to determine if the operationally relevant performance parameters (g-loads, final range-to-target and propellant usage) would yield acceptable values if the manual piloting task was simply to keep the steady-state bank angle errors within $6 \mathrm{deg}$. To limit propellant usage, pilots were advised not to correct errors under $2 \mathrm{deg}$. The experiment data showed that pilots were generally able to follow steady-state segments of the bank angle guidance command with an accuracy of 5 deg or better.

Range errors were always close to their corresponding benchmark autopilot values of $59.7 \mathrm{nmi}$ for the nominal case and $57.6 \mathrm{nmi}$ for the dispersed case. The mean range errors for the piloted formal evaluation runs were 60.3 $\mathrm{nmi}$ for the nominal case and $58.4 \mathrm{nmi}$ for the dispersed case, but the difference between the corresponding data sets was not statistically significant (P-value $>0.05)$. The individual range errors were all less than $70 \mathrm{nmi}$. A large range error (mainly cross-range) was expected for the loads-managed entry task because there were no bank reversals.

Propellant usage data showed a large spread relative to their corresponding benchmark autopilot values of 15 $\mathrm{lbm}$ for the nominal case and $31 \mathrm{lbm}$ for the dispersed case. The mean propellant usage values for the piloted formal evaluation runs were $41 \mathrm{lbm}$ for the nominal case and $68 \mathrm{lbm}$ for the dispersed case, and the propellant usage for piloted trajectories always exceeded the corresponding autopilot value. As expected, mean propellant usage for the dispersed case was substantially higher than that for the nominal case and the difference between the corresponding data sets was statistically significant (P-value $<0.05$ ). Propellant usage was less than $90 \mathrm{lbm}$ for $90 \%$ of the data points and always less than $120 \mathrm{lbm}$.

The above results indicate that the operationally relevant performance parameters (g-loads, final range-to-target and propellant usage) yield acceptable values for loads-managed entry if the manual piloting task is simply to keep the steady-state bank angle error within $\pm 6 \mathrm{deg}$. For the overall data set of nominal and dispersed cases, the mean range errors were within $1 \%$ of the benchmark autopilot values, while the mean propellant usage was roughly 2.5 times the benchmark autopilot values. This suggests that additional RCS propellant may need to be budgeted for this manual piloting contingency and that a looser guidance tracking limit may result in a better trade-off between range error and propellant usage.

Targeted Entry Task

Although this task had a steep learning curve, the pilots were able to develop a flying technique during the training period to meet the performance metrics. For this task, the performance parameters for the handling qualities assessment were range-to-target at drogue deployment, g-loads, and propellant usage.

The experiment data showed that $94 \%$ of the data points had range errors within desired the performance bound of $35 \mathrm{nmi}$ and all were well within the adequate performance bound of $70 \mathrm{nmi}$. For the h-dot schedule, the mean range errors were $23 \mathrm{nmi}$ for the nominal case and $16 \mathrm{nmi}$ for the dispersed case. For the full (h-dot, Mach, energy) 
schedule, the mean range errors were $16 \mathrm{nmi}$ for the nominal case and $14 \mathrm{nmi}$ for the dispersed case. Although the mean range error for the dispersed case was less than that for the nominal case for both types of schedules, the differences between the corresponding data sets were not statistically significant (P-value $>0.05$ ). Additionally, for both nominal and dispersed cases, the differences between range error data sets for $\mathrm{h}$-dot schedule and full schedule were not statistically significant (P-value $>0.05)$. This indicates that the open-loop schedule, obtained from a primary-guidance-following autopilot run in the nominal scenario, is quite robust to large dispersions in key parameters such as atmospheric conditions, capsule aerodynamics, c.m. location, RCS jet properties, and navigation errors.

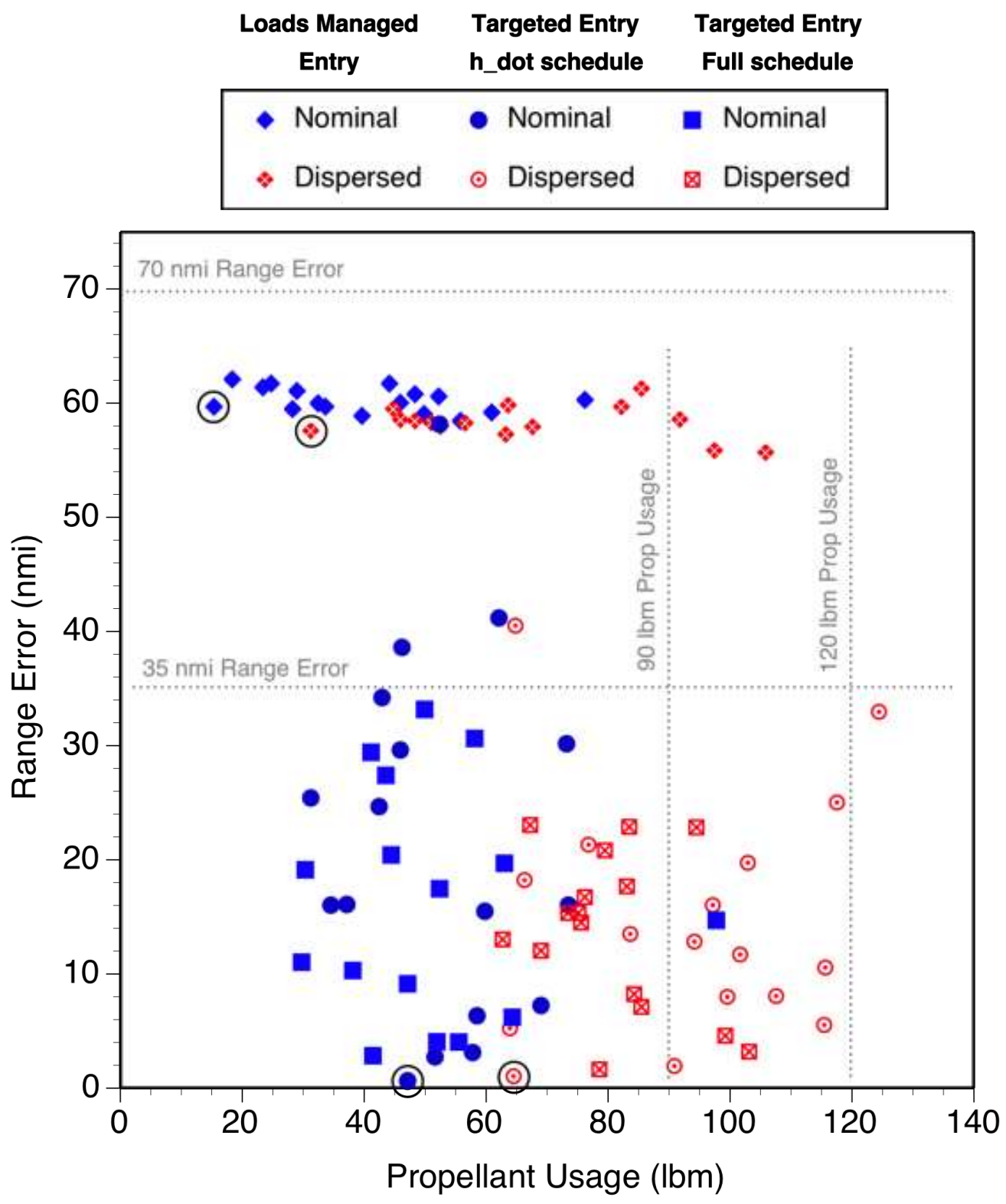

Figure 5. Range error and propellant usage (circles indicate autopilot data) 
Propellant usage was within the desired performance bound of $90 \mathrm{lbm}$ for $77 \%$ of the data points, with only $2 \%$ of the data outside the adequate performance bound of $120 \mathrm{lbm}$. For the h-dot schedule, the mean propellant usage was $52 \mathrm{lbm}$ for the nominal case and $95 \mathrm{lbm}$ for the dispersed case. For the full (h-dot, Mach, energy) schedule, the mean propellant usage was $51 \mathrm{lbm}$ for the nominal case and $81 \mathrm{lbm}$ for the dispersed case. As expected, for both types of schedules, mean propellant usage for the dispersed case was substantially higher than that for the nominal case and the difference between the corresponding data sets was statistically significant $(\mathrm{P}$-value $<0.05)$. For both nominal and dispersed cases, the differences between propellant usage data sets for $\mathrm{h}$-dot schedule and full schedule were not statistically significant (P-value $>0.05)$.

The above results indicate that the complex entry trajectory can be piloted using simple intuitive rules to follow an open-loop schedule, and this provides acceptable values of g-loads, final range-to-target and propellant usage. Since the difference between the range error data sets for nominal and dispersed cases was not statistically significant, it indicates that the open-loop schedule is robust to large dispersions in key parameters that influence entry trajectories. Overall, compared to loads-managed entry performance, targeted entry achieved a $71 \%$ lower range error at the expense of $27 \%$ more propellant usage primarily due to the use of bank reversals.

\section{Handling Qualities Ratings}

Handling qualities ratings for the six experiment configurations are presented in Fig. 6 . In this bubble chart, the size of the bubble for a rating value indicates the number of pilots who assigned that rating, and a star symbol indicates the median rating. Each experiment configuration was rated by eight pilots, except for the dispersed loadsmanaged entry configuration which was rated by seven pilots.

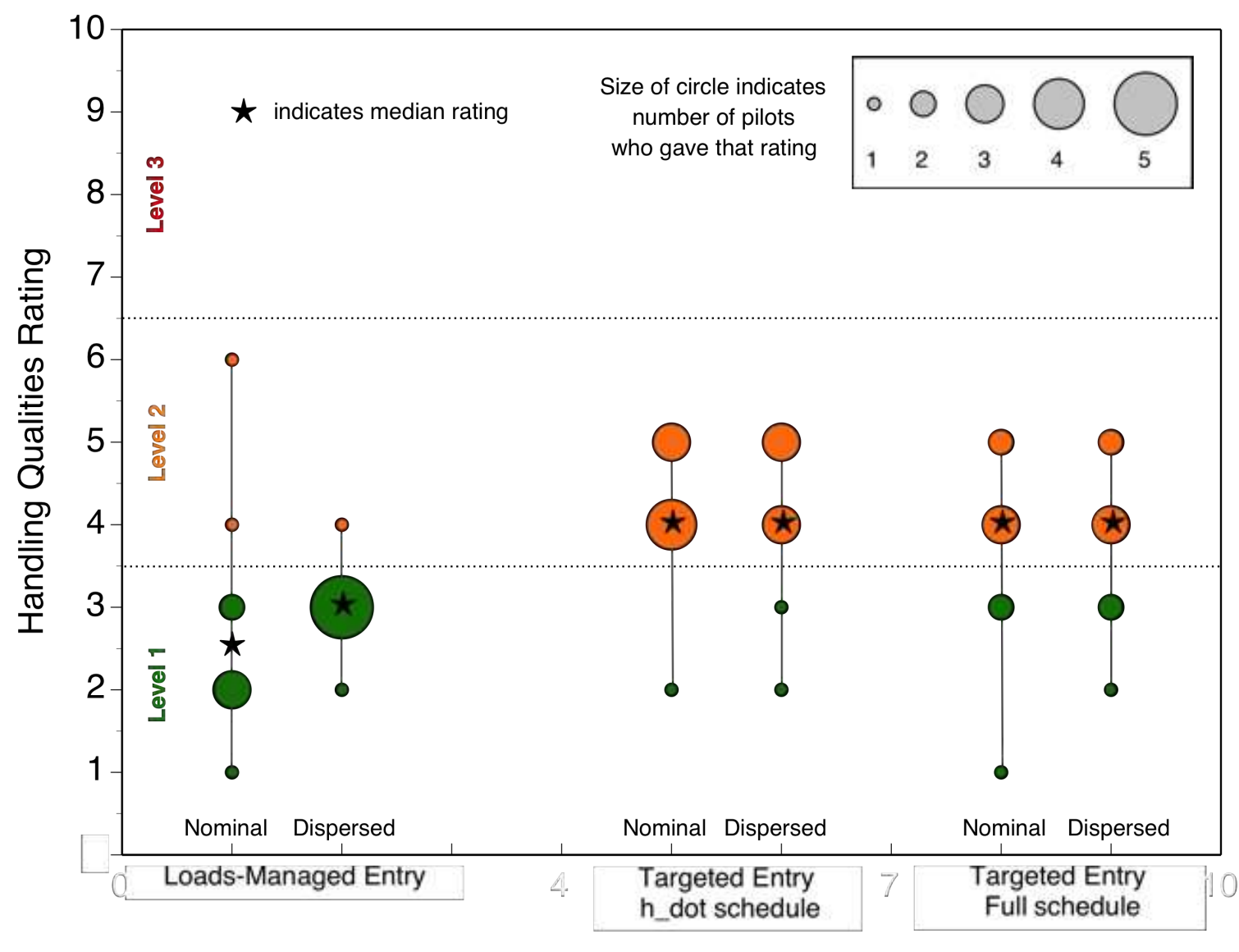

Piloting Task

Figure 6. Handling qualities ratings grouped by piloting task 


\section{Loads-Managed Entry Task}

The left portion of Fig. 6 shows data for nominal and dispersed cases for the loads-managed entry task. Most of the pilot ratings were Level 1, indicating satisfactory handling qualities. Pilot comments indicated that this was not a particularly challenging task. The primary, albeit not universal, complaint was about some high-frequency noise content in the guidance command which resulted in jittery movement of the error needle; this issue can be mitigated by filtering the guidance signal prior to display in the cockpit.

For the loads-managed entry task, the only performance parameter for the handling qualities assessment was steady-state bank angle error between the commanded and actual values, where desired and adequate performance bounds corresponded to error magnitudes less than $6 \mathrm{deg}$ and $10 \mathrm{deg}$ respectively. Desired performance was almost always achieved - the data were typically showed an accuracy of 5 deg or better. The handling qualities rating of 6 was assigned by a pilot who experienced mild oscillations while following the rather noisy guidance command too closely on one of his two formal evaluation runs for this configuration. This rating notwithstanding, the handling qualities ratings for the nominal configuration were slightly better than for the dispersed case.

\section{Targeted Entry Task}

The right portion of Fig. 6 shows data for nominal and dispersed cases for the two types of targeted entry tasks (h-dot schedule and full schedule). The pilot ratings were mostly Level 2, indicating that handling qualities warrant improvement. Suggestions for improvement included retaining data at the previous gate on the schedule display (in addition to data on current states and target values at the next gate), and displaying data on the primary display for acceleration along the local vertical (h-double-dot). Pilot comments indicated a preference for the option to use energy and/or Mach information in addition to h-dot information, and this is reflected by a slightly higher fraction of Level 1 ratings for the full schedule.

For the targeted entry tasks, the performance parameters were range-to-target at drogue deployment, g-loads, and propellant usage. The performance data presented in the previous sub-section indicated that pilots achieved desired performance most of the time. The Level 2 ratings can hence be attributed primarily to the pilot compensation required for determining the bank angle to follow the specified open-loop schedule. However, the noteworthy result for this task is not the Level 2 rating - it is the finding that such a complex piloting task can be accurately performed by using a simple open-loop schedule that is robust to large dispersions in key parameters such as atmospheric conditions, capsule aerodynamics, c.m. location, RCS jet properties, and navigation errors.

$\square$\begin{tabular}{c|cc|c|}
$\begin{array}{c}\text { Loads Managed } \\
\text { Entry }\end{array}$ & $\begin{array}{l}\text { Targeted Entry } \\
\text { Full schedule }\end{array}$ & $\begin{array}{l}\text { Targeted Entry } \\
h_{\text {_dot schedule }}\end{array}$
\end{tabular}

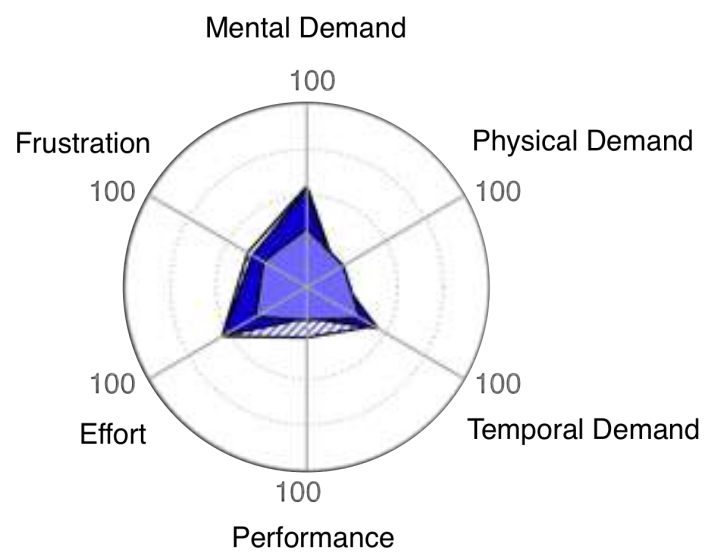

Nominal

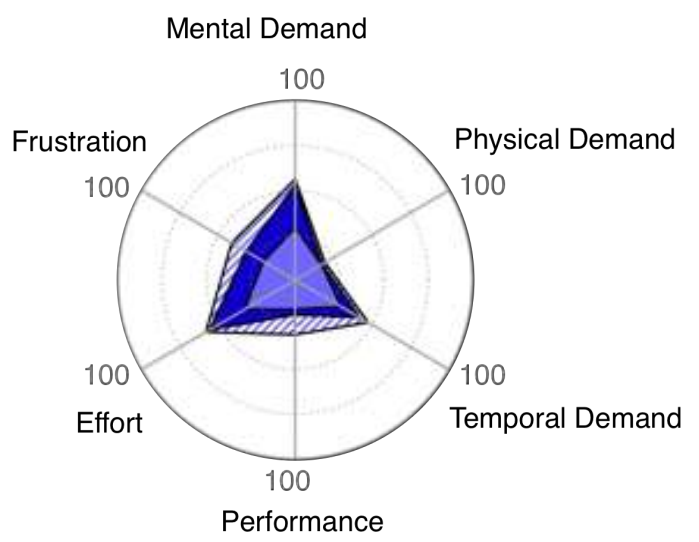

Dispersed

Figure 7. Task Load Index component ratings 


\section{Workload Ratings}

NASA Task Load Index (TLX) component ratings for the six experiment configurations, averaged across all pilots, are presented in Fig. 7. The data show that the primary TLX components for this flying task were mental demand, temporal demand, and effort, while the secondary components were physical demand, performance, and frustration. The loads-managed entry task had the lowest workload components, and for targeted entry tasks the full schedule had slightly lower workload components than the h-dot schedule; this was true for both nominal and dispersed cases. For each of the three piloting tasks, there were only minor differences between the nominal and dispersed cases.

\section{Conclusions}

An evaluation of handling qualities for atmospheric capsule entry was conducted by eight pilots, including six astronauts, flying the NASA Ames Vertical Motion Simulator. The piloting tasks in this experiment were a bank angle guidance command tracking task for loads-managed entry, and two types of flight parameter (vertical speed, Mach, energy) schedule following tasks for targeted entry. Each piloting task included two scenarios featuring nominal values and high dispersions for key parameters such as atmospheric conditions, capsule aerodynamics, c.m. location, RCS jet properties, and navigation errors. Performance parameters of operational interest were range-totarget at drogue deployment, g-loads, and RCS propellant usage. In all cases, the g-loads were found to be within human endurance limits.

For the loads-managed entry task, the pilot followed closed-loop bank angle guidance commands generated by the backup loads-managed guidance system. The piloting task was to correct the error between the actual and commanded bank angles based on visual cues presented on the cockpit displays. Pilots were able to track steadystate segments of the guidance commands with an accuracy of 5 deg or better. With this level of tracking accuracy, the mean range-to-target values at drogue deployment were within $1 \%$ of the benchmark autopilot values, while the mean propellant usage was roughly 2.5 times the benchmark autopilot values. These results indicate that additional RCS propellant may need to be budgeted for this manual piloting contingency, and that a looser guidance tracking limit may result in a better trade-off between range error and propellant usage. Overall, the handling qualities ratings for task were Level 1 . Pilot comments indicated that this was not a particularly challenging task.

For the targeted entry task, the pilot used simple intuitive rules to determine the desired bank angle, based on an open-loop schedule of certain flight parameters specified at several range-to-target gates along the entry trajectory. The flight parameters were vertical speed, Mach, and total energy. There were two types of schedule: a schedule for vertical speed only (h-dot schedule), and the full schedule of vertical speed, Mach, and energy. The piloting task was to determine bank angle adjustments by using schedule data presented on the cockpit displays and applying intuitive rules, e.g., raise lift vector if sinking too fast. Although there was a steep learning curve for this task, the pilots were able to develop a flying technique during the training period and get good scores on the performance metrics. Since this task included bank reversals, the range error was about $71 \%$ lower than that for the loadsmanaged entry task at the expense of about $27 \%$ higher propellant usage. Overall, the handling qualities ratings for this task were Level 2. Since desired performance was achieved most of the time, the Level 2 ratings can be attributed primarily to the pilot compensation required for determining the bank angle to follow the specified openloop schedule. Pilot comments for improvement include displaying schedule information for the previous gate and displaying data for acceleration along the local vertical (h-double-dot). The ratings were slightly better for the full schedule and pilot comments indicated a preference for the option to use energy and/or Mach information in addition to h-dot information. The noteworthy result for the targeted entry task is not the Level 2 ratings - it is the finding that such a complex piloting task can be performed accurately using a simple open-loop schedule that is robust to large dispersions.

\section{Acknowledgments}

The efforts of the VMS staff at NASA Ames are greatly appreciated. In particular, the authors would like to acknowledge the substantial contributions of Mike Weinstein, Mike Leonard, Scott Reardon and Nghia Vuong who developed the simulation software and also served as simulation engineers for the experiment. Bo Bobko served as project pilot and contributed to model development and testing. The authors are grateful to the members of the Orion Entry MODE Team at the Johnson Space Center for conducting the Monte Carlo analysis that provided a foundation for determining desired/adequate bounds for key performance parameters. 


\section{References}

${ }^{1}$ Cooper, G.E. and Harper, R.P., "The Use of Pilot Rating in the Evaluation of Aircraft Handling Qualities," NASA TN D5153, April 1969.

${ }^{2}$ Soulé, H.A., "Preliminary Investigation of the Flying Qualities of Airplanes," NACA TR 700, 1940.

${ }^{3}$ Gilruth, R.R., "Requirements for Satisfactory Flying Qualities of Airplanes," NACA TR 755, 1941.

${ }^{4}$ Harper, R.P. and Cooper G.E, "Handling Qualities and Pilot Evaluation," Journal of Guidance, Control, and Dynamics, Vol. 9, No. 5, Sep-Oct 1986, pp. 515-529.

${ }^{5}$ Phillips, W.H., "Flying Qualities from Early Airplanes to the Space Shuttle," Journal of Guidance, Control, and Dynamics, Vol. 12, No. 4, 1989, pp. 449-459.

${ }^{6}$ Mitchell, D.G., Doman, D.B., Key, D.L., Klyde, D.H., Leggett, D.B., Moorhouse, D.J., Mason, D.H., Raney, D.L, and Schmidt, D.K., "Evolution, Revolution, and Challenges of Handling Qualities," Journal of Guidance, Control, and Dynamics, Vol. 27, No. 1, 2004, pp. 12-28.

7"Military Specification, Flying Qualities of Piloted Airplanes," U.S. Air Force, MIL-F-8785C, Wright-Patterson Air Force Base, OH, Nov. 1980.

"“Aeronautical Design Standard, Performance Specification: Handling Qualities Requirements for Military Rotorcraft,” U.S. Army - Aviation and Missile Command, ADS-33E-PRF, Redstone Arsenal, AL, March 2000.

${ }^{9}$ Meeker, J.I., "Evaluation of Lateral-Directional Handling Qualities of Piloted Re-Entry Vehicles Utilizing Fixed-Base and In-Flight Evaluations," NASA CR-778, May 1967.

${ }^{10}$ Wingrove, R.C. , Stinnett, G.W., and Innis, R.C., “A Study of the Pilot's Ability to Control an Apollo Type Vehicle During Atmosphere Entry," NASA TN-2467, August 1964.

${ }^{11}$ Graves, C.A. and Harpold, J.C., "Apollo Experience Report - Mission Planning for Apollo Entry,” NASA TN-6725, March 1972.

${ }^{12}$ Frank, A.J., Knotts, E.F., and Johnson, B.C., “An Entry Monitor System for Maneuverable Vehicles,” Journal of Spacecraft and Rockets, Vol. 3, No. 8, August 1966, pp. 1229-1234.

${ }^{13}$ NASA's Exploration Systems Architecture Study, NASA TM-2005-214062, November 2005.

${ }^{14}$ Sim, L., Cummings, M.L., and Smith, C.A., "Past, Present and Future Implications of Human Supervisory Control in Space Missions," Acta Astronautica, Vol. 62, 2008, pp. 648-655.

${ }^{15}$ Anon, "Human-Rating Requirements for Space Flight Systems," NASA Procedural Requirements, NPR 8705.2B, May 2008, pp. 34-35.

${ }^{16}$ Bailey, R.E., Jackson, E.B., Bilimoria, K.D., Mueller, E.R., Frost, C.R., and Alderete, T.S., “Cooper-Harper Experience Report for Spacecraft Handling Qualities Applications,” NASA TM-2009-215767, June 2009.

${ }^{17}$ Bilimoria, K.D., "Effects of Control Power and Guidance Cues on Lunar Lander Handling Qualities," Journal of Spacecraft and Rockets, Vol. 46, No. 6, November-December 2009, pp. 1261-1271.

${ }^{18}$ Mueller, E., Bilimoria, K.D., and Frost, C., "Effects of Control Power and Inceptor Sensitivity on Lunar Lander Handling Qualities," Paper No. 2009-6407, AIAA Space 2009 Conference, September 2009.

${ }^{19}$ Mueller, E., Bilimoria, K.D., and Frost, C., "Improved Lunar Lander Handling Qualities through Control Response Type and Display Enhancements," Paper No. 2010- 8025, AIAA Guidance, Navigation, and Control Conference, August 2010.

${ }^{20}$ Bailey, R., Jackson, B., Goodrich, K., Ragsdale, A., Neuhaus, J., and Barnes, J., "Investigation of Reaction Control System Design on Spacecraft Handling Qualities for Docking," Journal of Guidance, Control, and Dynamics, Vol. 32, No. 6, NovemberDecember 2009, pp. 1723-1735.

${ }^{21}$ Mueller, E., Bilimoria, K.D., and Frost, C., "Dynamic Coupling and Control Response Effects on Spacecraft Handling Qualities during Docking," Journal of Spacecraft and Rockets, Vol. 46, No. 6, November-December 2009, pp. $1288-1297$.

${ }^{22}$ Bilimoria, K.D., Mueller, E., and Frost, C., "Handling Qualities Evaluation of Pilot Tools for Spacecraft Docking in Earth Orbit,” Paper No. 2009-5665, AIAA Guidance, Navigation, and Control Conference, August 2009.

${ }^{23}$ Hart, S.G. and Staveland, L.E., "Development of NASA-TLX (Task Load Index): Results of Empirical and Theoretical Research," in Human Mental Workload, P.A. Hancock and N. Meshkati (Eds.), North Holland Press, Amsterdam, The Netherlands, 1988, pp. 139-183.

${ }^{24}$ Acevedo, A., Arnold, J., and Othon, W., "ANTARES: Spacecraft Simulation for Multiple User Communities and Facilities," Paper No. 2007-6888, AIAA Modeling and Simulation Technologies Conference, August 2007.

${ }^{25}$ Gay, R.S., "NASA ANTARES Simulation GNC Architecture," Paper No. 2007-6679, AIAA Modeling and Simulation Technologies Conference, August 2007.

${ }^{26}$ Broome, J.R. and Johnson, W., "Orion Entry, Descent, and Landing Performance and Mission Design,” Paper No. 20076430, AIAA Modeling and Simulation Technologies Conference, August 2007.

${ }^{27}$ Hoelscher, B.R., "Orion Entry, Descent, and Landing Simulation," Paper No. 2007-6428, AIAA Modeling and Simulation Technologies Conference, August 2007.

${ }^{28}$ Roscoe, A.H. and Ellis, G.A., "A Subjective Rating Scale for Assessing Pilot Workload in Flight: A Decade of Practical Use," Royal Aerospace Establishment (RAE) Technical Report TR 90019, 1990.

${ }^{29}$ Danek, G.L., "Vertical Motion Simulator Familiarization Guide," NASA TM 103923, May 1993.

${ }^{30}$ Aponso, B.L., Tran, D.T., Schroeder, J.A., and Beard, S.D., "Rotorcraft Research at the NASA Vertical Motion Simulator," Paper 2009-6056, AIAA Atmospheric Flight Mechanics Conference, August 2009. 